\title{
Brief Note on a Scalar Quantum Field with Finite Lifetime in a Lorentz Invariant Non-Rectangular Euclidean Space
}

\author{
Carmen Tornow \\ Institute of Planetary Research, DLR, Berlin, Germany \\ Email: carmen.tornow@dlr.de
}

Received 17 April 2014; revised 14 May 2014; accepted 11 June 2014

Copyright (C) 2014 by author and Scientific Research Publishing Inc. This work is licensed under the Creative Commons Attribution International License (CC BY). http://creativecommons.org/licenses/by/4.0/

\section{(c) (i) Open Access}

\begin{abstract}
A not necessary rectangular Euclidean space (NoNRES) is constructed, in which one obtains a generally Lorentz invariant scalar product for the low energy sector (LES). This sector is defined for energies below the Planckian limit. If the energy is zero, the NoNRES becomes rectangular and due to the Lorentz invariance, it is applicable for the complete LES of the theory. In contrast to the usual Minkowski space the metric of the NoNRES depends on the kinetic energy of the observed quantum particles. It is assumed that this metric may be useful to derive the scattering cross-section of the corresponding quantum field theory. This assumption is related to the occurrence of divergent loop momentum integrals caused by including the infinite energy range above the Planckian limit (high energy sector or HES). Due to its energy dependence, the metric in both energy sectors differs. In the HES, it depends on the effective dimension of the NoNRES. This dependency results from fluctuations of the space-time above the Planckian limit. Even if they are not part of the theory (as they would be in quantum gravity), these fluctuations should not be ignored. The effective dimension decreases if the energy of the considered particle increases. Since this is true for the HES only, the ultraviolet divergences of loop integrals seem to vanish without distorting the results of the LES. The mechanism is illustrated by calculating the tadpole integral occurring for a simple self-interacting scalar quantum field (with the Higgs mass as example). One obtains a finite contribution for the integral and consequently for the lifetime of the scalar particle.
\end{abstract}

\section{Keywords}

Lorentz Invariance, Scalar Field, Renormalisation, Lifetime

\section{Introduction}

Ultraviolet (UV) divergences in quantum field theories (QFTs) without regularization and renormalization occur 
because of short-distance interactions between the elementary particles. These interactions are considered in the high energy range of loop-momentum integrals. A well-known example for this behavior is the one loop-momentum integral of the scalar QFT with a $\varphi^{4}$ potential term to simulate self-interacting. Although, this theory must be seen as an artificial test model, we know that a scalar field is related to the Higgs boson [1] [2]. If one assumes a very small coupling constant for the $\varphi^{4}$ potential, the dressed propagator $\Delta\left(x_{1}, x_{2}\right)$ is given by the one-loop approximation and consists of the free field propagator $D\left(x_{1}, x_{2}\right)$, and the tadpole diagram (Figure 1 ).

An analysis of the momentum integral corresponding to the tadpole diagram shows that its divergence degree is +2 . In order to calculate the corrected mass and lifetime of the self-interacting scalar field a regularization and renormalization procedure is needed. In general, the divergences in loop-momentum integrals originate from the fact, that special QFTs, e.g. quantum electrodynamics, are low energy approximations of a complete theory of all possible quantum particle interactions, including gravity [3]. In this study it is asked whether there is chance to simplify the regularization and renormalization procedure. As a first step to seek an answer to this question, the upper dressed propagation operator defined in Figure 1 is computed with a modified method. This method requires a split of the integration range using the Planckian mass, $m_{P}=E_{P} / c^{2}$ where $E_{P}=1.22 \times 10^{19} \mathrm{GeV}$ is the Planck energy. The related split value is $k_{P}=\left(m_{P} c / \hbar\right)^{2}$. Accordingly, one gets for the spatial frequency $k=$ $2 \pi / \lambda$ or if we consider the particle masses of real and virtual particles $k=2 \pi l$ where $l$ is the inverse Compton length $l=m_{0} \cdot c / \hbar$,

- a low energy sector (LES) with $k^{2}<k_{P}^{2}$, i.e. Lorentz invariance of measurable (scalar) values, and

- a high energy sector (HES) with $k^{2} \geq k_{P}^{2}=\left(m_{P} c / \hbar\right)^{2}$, i.e. Lorentz symmetry is broken.

Actually, between the energy sector of standard QFT and the HES an appropriate transition region might exist, since it is speculated that the effects related to the violation of Lorentz invariance (e.g. polarization independent refractivity [4]) can already be detected at energies much lower than $E_{P}=m_{P} c^{2}$. Nonetheless, it will be shown in section 3 that for the simple scalar quantum field a sharp separation works well.

Because an interval split is useless if one integrates over both energy intervals with the same method, an integration measure needs to be derived that differs between LES and HES. This measure shall reflect the lower retrievable information content of the HES compared to the LES. The next two sections explain how this measure is derived and implemented mathematically. In Section 4, the modified method of computing loop integrals is applied to the tadpole integral illustrated in Figure 1. Lastly, the applicability of this integration method is discussed and further steps to establish this technique are outlined.

\section{Invariance of Lorentz Transformation in the Non-Rectangular Euclidean Space}

It is assumed that the integration measure directly related to the geometry of Minkowski space (MS) may not be appropriate for the calculation of the high energy range of momentum integrals. Therefore, an alternative, Lorentz invariant, geometry is constructed. It turns out that a non-rectangular space with Euclidean signature and an energy dependent angle between its space and time axes can be used. This non-rectangularity is useful to obtain an energy dependent integration measure. In this space, which is abbreviated by NoNRES (see section 3), one has general transformations given by

$$
\begin{array}{lll}
x_{0}^{\prime}=\omega_{j}\left(x_{0}-\sigma_{j} x_{j}\right) & \text { and } & x_{i}^{\prime}=\omega_{j}\left(x_{i}-\sigma_{j} x_{0}\right) \\
x_{0}=\omega_{j}\left(x_{0}^{\prime}+\sigma_{j} x_{j}^{\prime}\right) & \text { and } & x_{i}=\omega_{j}\left(x_{i}^{\prime}+\sigma_{j} x_{0}^{\prime}\right) .
\end{array}
$$

between an inertial system $\Sigma$ moving with the velocity $\boldsymbol{v}=V_{j} \boldsymbol{e}_{j}$ and an inertial system $\Sigma$ ' moving with a velocity $\boldsymbol{v}^{\prime}=V_{j}^{\prime} \boldsymbol{e}_{j}$. The index $j$ is arbitrary but fixed. Further one sets $x_{0}=c t \neq x_{0}^{\prime}=c t^{\prime}, x_{j} \neq x_{j}^{\prime}, \quad x_{k}=x_{k}^{\prime}$ for $k \neq j$. As for the MS the scalar product of vector $x=\left(x_{0}, x_{1}, x_{2}, x_{3}\right)^{\mathrm{T}}$ is Lorentz invariant, i.e.,

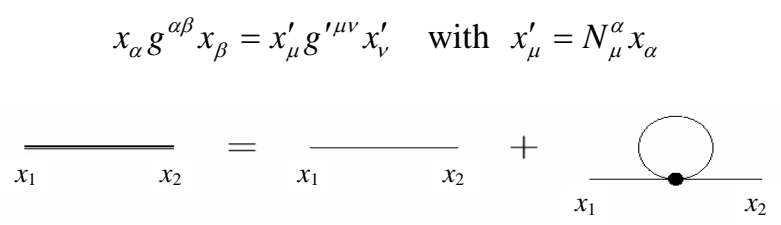

Figure 1. Two Feynman diagrams (right-hand side) giving the first order dressed propagator (left-hand side) of the weakly self-interacting scalar field, $\varphi$. 
where the matrix $N_{\alpha}^{\mu}$ describes the Lorentz transformation (LT) and $g^{\alpha \beta}\left(g^{\prime \mu \nu}\right)$ is the metric tensor in the NoNRES. From Equation (2) we obtain

$$
g^{\alpha \beta}=N_{\mu}^{\alpha} g^{\prime \mu v} N_{v}^{\beta} \quad \text { and } \quad N_{\alpha}^{\mu} N_{v}^{\alpha}=\delta_{v}^{\mu} .
$$

The parameter $\sigma_{j}$ depend on the velocity ratios $\beta_{j}=V_{j} / c$ and $\beta_{j}^{\prime}=V_{j}^{\prime} / c$ where $j=1,2,3$ is arbitrary but fixed. If one assumes for $\sigma_{j}$ and $\omega_{j}$ a relation typical for the LT, all the non-zero elements of $N_{\alpha}^{\mu}$ can be written according to Equation (1) in the following way

$$
N_{0}^{0}=N_{j}^{j}=\omega_{j}, \quad N_{k}^{k}=1, \quad N_{0}^{j}=N_{j}^{0}=-\sigma_{j} \omega_{j} \quad \text { where } \omega_{j}=\left|1-\sigma_{j}^{2}\right|^{-1 / 2} \text { and } k \neq j .
$$

All off-diagonal elements of the metric $g^{0 j}\left(g^{\prime 0 j}\right)$ in the NoNRES are related to the angle between the axis $x_{j}$ $\left(x_{j}^{\prime}\right)$ and $x_{0}\left(x_{0}^{\prime}\right)$, which is influenced by the ratio $\beta_{j}\left(\beta_{j}^{\prime}\right)$. Based on these assumptions one obtains for the elements of $g^{\mu \nu}$

$$
g^{00}=g^{j j}=\lambda_{j}^{-1}, \quad g^{k k}=1, \quad g^{j 0}=g^{0 j}=-\rho_{j} \lambda_{j}^{-1} \quad \text { where } \lambda_{j}=\sqrt{1-\rho_{j}^{2}} \text { and } k \neq j .
$$

The equivalent approach follows for the metric $g^{\prime \mu \nu}$ in $\Sigma^{\prime}$ using the coefficients $\rho_{j}^{\prime}$ and $\lambda_{j}^{\prime}$. When $\Sigma$ is an inertial system for a resting body its metric $g^{\mu v}=\delta^{u v}$ with $\delta^{u v}=1$ if $\mu=v$ and $\delta^{u v}=0$ if $\mu \neq v$. An expression that relates $\rho_{j}$ and $\rho_{j}^{\prime}$ to $\sigma_{j}$ can be derived inserting the Equations (4) and (5) into Equation (3):

$$
\left(N_{0}^{0}\right)^{2}-2 \rho_{j}^{\prime} N_{0}^{0} N_{j}^{0}+\left(N_{j}^{0}\right)^{2}=\frac{\sqrt{1-\rho_{j}^{\prime 2}}}{\sqrt{1-\rho_{j}^{2}}} \text { and } \rho_{j}^{\prime}\left(N_{0}^{0}\right)^{2}-2 N_{0}^{0} N_{j}^{0}+\rho_{j}^{\prime}\left(N_{j}^{0}\right)^{2}=\rho_{j} \frac{\sqrt{1-\rho_{j}^{\prime 2}}}{\sqrt{1-\rho_{j}^{2}}} \text {. }
$$

Because the absolute value $\left|1-\sigma_{j}^{2}\right|$ occurs in Equation (4) there are two solutions for Equation (6)

$$
\sigma_{j}=\left(\rho_{j}^{\prime} \lambda_{j}-\rho_{j} \lambda_{j}^{\prime}\right) /\left(\lambda_{j}+\lambda_{j}^{\prime}\right) \text { or } \hat{\sigma}_{j}=\left(\rho_{j}^{\prime} \lambda_{j}+\rho_{j} \lambda_{j}^{\prime}\right) /\left(\lambda_{j}-\lambda_{j}^{\prime}\right) .
$$

We note that $\sigma_{j}<1$ and $\hat{\sigma}_{j}>1$. The relations in Equation (7) correspond to the addition theorems of the velocity ratios $\beta_{j}$ and $\beta_{j}^{\prime}$. To show this, we need to express $\sigma_{j}$ and $\hat{\sigma}_{j}$ by $\beta_{j}\left(\beta_{j}^{\prime}\right)$. It follows from Equation (5) that $\rho_{j}=0$ and $\lambda_{j}=1$ for a system at rest, $\Sigma$. Equation (7) simplifies to

$$
\sigma_{j,+}^{0}=\rho_{j}^{\prime} /\left(1+\lambda_{j}^{\prime}\right) \text { and } \hat{\sigma}_{j,+}^{0}=\rho_{j}^{\prime} /\left(1-\lambda_{j}^{\prime}\right)
$$

where the index 0 indicates that one inertial system, $\Sigma$, is at rest. The two plots in Figure 2 present the inverse function, i.e., the curves of $\rho_{j}^{\prime}$ and $\lambda_{j}^{\prime}$ versus the parameters $\sigma_{j,+}$ and $\hat{\sigma}_{j,+}$. It is interesting to recognize the increase of $\rho_{j}^{\prime}$ on the left-hand plot and the decrease of $\rho_{j}^{\prime}$ on the right-hand plot.

This behaviour ensures that the metric given by Equation (5) is real. Now, $\Sigma^{\prime}$ is at rest and $\Sigma$ is the inertial system for a moving body. We set $\rho_{j}^{\prime}=0, \quad \lambda_{j}^{\prime}=1$ in Equation (7) and obtain
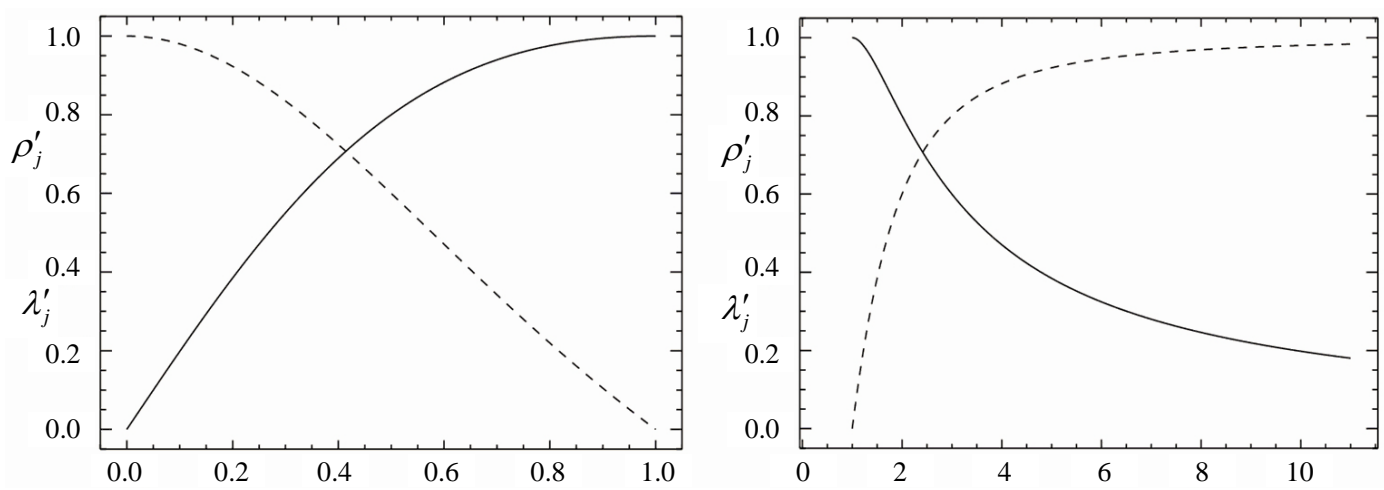

Figure 2. Influence of velocity ratio $\sigma_{j,+}^{0}$ and $\hat{\sigma}_{j,+}^{0}$ (abscissa) on metric parameter $\rho_{j}^{\prime}$ (solid line) and $\lambda^{\prime}$ (dashed line) for $V_{j}^{\prime}<c$ and $V_{j}^{\prime}>c$. 


$$
\sigma_{j,-}^{0}=-\rho_{j} /\left(1+\lambda_{j}\right) \quad \text { and } \quad \hat{\sigma}_{j,-}^{0}=-\rho_{j} /\left(1-\lambda_{j}\right) .
$$

Inserting Equations (8.1) and (8.2) into the relation for $\sigma_{j}$ and $\hat{\sigma}_{j}$ in Equation (7) it can be shown that

$$
\begin{aligned}
& \sigma_{j}=\left(\sigma_{j,+}^{0}+\sigma_{j,-}^{0}\right) /\left(1+\sigma_{j,+}^{0} \sigma_{j,-}^{0}\right) \\
& \hat{\sigma}_{j}=\left(1+\hat{\sigma}_{j,+}^{0} \hat{\sigma}_{j,-}^{0}\right) /\left(\hat{\sigma}_{j,+}^{0}+\hat{\sigma}_{j,-}^{0}\right)
\end{aligned}
$$

which are indeed the addition theorems of the velocity ratios known from special relativity in the MS, where parameters $\sigma_{j,+}^{0}$ and $\sigma_{j,-}^{0}$ are equal to $\beta_{j}$ and $\beta_{j}^{\prime}$, while for $\hat{\sigma}_{j,+}^{0}$ and $\hat{\sigma}_{j,-}^{0}$ one finds $\hat{\beta}_{j}=1 / \beta_{j}$ and $\hat{\beta}_{j}^{\prime}=1 / \beta_{j}^{\prime}$. Note, that matrix $N_{\alpha}^{\mu}$ is identical to the LT. In addition, time- and space-like regions can be identified in the NoNRES, which are related to both of the solutions in Equation (7). The first relation in Equations (7) and (9) is the addition theorem of velocities lower than $c$ and the second relation is the addition theorem of velocities higher than $c$. The index $j$, that can have values between 1 and 3 , gives the three matrices $N_{\alpha}^{\mu}(j)$ in Equation (4) that form the Lorentz group in the NoNRES. In special relativity, a fundamental parameter is the "Eigenzeit" following from the approach

$$
\mathrm{d} s^{2}=g^{\mu v} \mathrm{~d} x_{\mu} \mathrm{d} x_{v}=\left(\mathrm{d} x_{0}^{2}+\mathrm{d} x_{j}^{2}-2 \rho_{j} \mathrm{~d} x_{0} \mathrm{~d} x_{j}\right) / \lambda_{j}=c^{2} \mathrm{~d} t_{e}^{2} \quad \text { with } \mathrm{d} x_{k}^{2}=0, k \neq j .
$$

It agrees with the "Eigenzeit" in the MS and verifies the identity between $\sigma_{j}^{0}$ and $\beta_{j}$ (the plus index is suppressed)

$$
\mathrm{d} t_{e}^{2}=\mathrm{d} t^{2}\left[1-\left(\sigma_{j}^{0}\right)^{2}\right] \Rightarrow \sigma_{j}^{0}=\beta_{j} \leq 1
$$

Using this "Eigenzeit” the energy-mass relation can be derived. For the particle energy $E$ we obtain

$$
E=c m_{0} u_{0}=c m_{0} \omega_{j} \dot{x}_{0}=\frac{m_{0} c^{2}}{\sqrt{1-\left(\sigma_{j}^{0}\right)^{2}}} \equiv m c^{2} \text { with } \lambda_{j}=\frac{1-\left(\sigma_{j}^{0}\right)^{2}}{1+\left(\sigma_{j}^{0}\right)^{2}} \text { and } \rho_{j}=\frac{2 \sigma_{j}^{0}}{1+\left(\sigma_{j}^{0}\right)^{2}}
$$

where the vector $\mathbf{u}=\left(\mathrm{d} x_{0} / \mathrm{d} t_{e}, \mathrm{~d} x_{1} / \mathrm{d} t_{e}, \mathrm{~d} x_{2} / \mathrm{d} t_{e}, \mathrm{~d} x_{3} / \mathrm{d} t_{e}\right)$ is the four-velocity, and the vector $\mathbf{p}=m_{0} \mathbf{u}$ is the four-momentum, if the particle rest mass is $m_{0}$. The energy-mass relation in Equation (13) is well-known from special relativity in the MS. Using this equation we express the metric parameter $\lambda_{j}$ as

$$
\lambda_{j}=m_{0}^{2} c^{4} /\left(2 E^{2}-m_{0}^{2} c^{4}\right)=\sqrt{1-\rho_{j}^{2}}
$$

For a particle nearly at rest with $E \sim m_{0} c^{2}$, the NoNRES becomes almost rectangular, i.e., $\lambda_{j} \sim 1$ and $\rho_{j} \sim 0$. From Equation (14) it is clear that the angle between the time and the relevant space axis in $\Sigma$ depends on the particle energy, just as required at the beginning of this section. If the particle energy of the quantum field influences the metric attached to it, the NoNRES cannot be globally an Euclidean space, $\mathbf{R}^{n}$, where $n=4$. It seems rather to be a manifold, $\mathbf{M}^{n}$, that is locally isomorphic to $\mathbf{R}^{n}$. The corresponding local positions in the NoNRES are infinitesimal small regions in space and time given by the interaction events between the quantum fields.

In the following section we derive the effective dimension of the NoNRES and describe the consequences of the geometry for this number. The effective dimension is a key ingredient for the desired integration measures allowing a low-weighted consideration of the high energy sector.

\section{The Effective Dimension of the NoNRES}

Using Equation (2) we can transform any inertial system in the NoNRES into a rectangular Euclidean space. However, doing so requires a continuous space-time with a well-defined metric. For energies in the Planckian range space-time fluctuations occur [4] (which may cause the polarization independent refractivity [5]) and this condition is not fulfilled. Since Equation (2) can be understood as a symmetry, where the related group is the Lorentz group, the emergence of the non-continuous space-time structure leads to a violation of Equation (2) and thus to a broken Lorentz symmetry. In the upper energy range of the LES and in the HES fluctuations have to be expected, if a virtual (off-shell) particle with an energy near the Planckian range interacts with a real 
(on-shell) or another virtual particle. They cause uncertainties for the physical parameters containing expressions like $g^{\mu v} k_{\mu} k_{\nu}$ or with similar terms. Because of Equation (13) the value of $\rho_{j}$ approaches 1 if the particle energy tends to infinity. Accordingly, the angle between the space and time axis decreases to $0^{\circ}$. Moreover, all the other spatial coordinate ranges are correspondingly shrunk compared to the ranges of $x_{0}$ and $x_{j}$, since the factor $\lambda_{j}^{-1}=\left(1-\rho_{j}^{2}\right)^{-1 / 2}$ tends to infinity. As a result, the information on the dynamics of the physical parameters is overwhelmed by the metric fluctuations. Due to a broken Lorentz symmetry caused by the non-continuous space-time, the non-rectangularity of the NoNRES becomes manifest in the HES. In the LES it appears to be a transformation effect which does not seriously destroy the accuracy of the theory. Consequently all scalar values can be calculated for a 4-dimensional rectangular space in the LES. In order to consider this geometrical behaviour of our special type of space-time in its name, it is called not necessary rectangular Euclidean space. The information loss for real high quality measurements (i.e. measurements with finite but sufficient accuracy) can be quantified. An appropriate measure to describe the mixing degree of quantum states is von Neumann's entropy $S_{N}$ given by [6]

$$
S_{N}(\phi)=-k_{B} \operatorname{Trace}[\phi \log (\phi)] \text { with } \phi_{k l}=\left|\theta_{k}\right\rangle\left\langle\theta_{l}\right|
$$

where $\varphi$ is the density matrix of an ensemble of quantum mechanical states $\theta_{k}$. Assuming that the density matrix fulfils the eigenvalue equations: $\boldsymbol{\phi} \boldsymbol{u}_{\alpha}=\boldsymbol{e}_{\alpha} \boldsymbol{u}_{\alpha}$, we can simplify Equation (15) an write:

$$
S_{N}(\phi)=-k_{B} \Sigma e_{\alpha} \log \left(e_{\alpha}\right) \text { with } 1=\Sigma e_{\alpha}
$$

where $\boldsymbol{e}_{\alpha}$ and $\left(\boldsymbol{u}_{k}\right)_{\alpha}$ are the eigenvalues and -vectors of the $d \times d$ density matrix $\boldsymbol{\phi}$. If the $d$ eigenvalues $\left\{e_{\alpha}\right\}$ with $\alpha=0, d-1$ are normalized as given in Equation (16) they can be linked to the probabilities of the states $\theta_{k}$. Then, the von Neumann entropy in Equation (16) agrees with Shannon's entropy and the related density matrix represents an ensemble of pure states. If one sets for $\phi$ the metric $g$ of the NoNRES, Shannon's entropy seems to be useful to derive an effective dimension, $\operatorname{dim}(g)$, for the NoNRES even if $g$ in Equation (5) depends on classical observables and not on the equivalent quantum operators (compare to Equation (14)). Using the entropy in Equation (16), one computes the effective dimension $\operatorname{dim}(g)$ by the following expression

$$
\operatorname{dim}(g)=\exp \left\{S_{N}(g) / k_{B}\right\}=\exp \left\{-\sum_{\alpha=0}^{3} \bar{e}_{\alpha}[g(E)] \ln \left(\bar{e}_{\alpha}[g(E)]\right)\right\} \quad \text { and } \quad 1=\sum_{\alpha=0}^{3} \bar{e}_{\alpha}[g(E)]
$$

where the bar indicates normalization of the eigenvalues of the metric. The notation $\operatorname{dim}[g(E)]=: \operatorname{dim}(E)$ is used to underline the energy dependency of the effective dimension which is caused by the energy dependency of the metric. If $\operatorname{dim}(E)=4$, the information content of the four vector components is measurable without any loss due to the finite accuracy of the measured data. If $\operatorname{dim}(E)<4$ uncertainties resulting from measurement errors can increase up to infinity if the smallest eigenvalue approaches zero [7]. According to Equation (5) the eigenvalues of the metric in their normalized form are

$$
\bar{e}_{0,1}(E)=\left(1 \pm \rho_{j}\right) /\left[2\left(1+\lambda_{j}\right)\right] \text { and } \bar{e}_{2,3}(E)=\lambda_{j} /\left[2\left(1+\lambda_{j}\right)\right] .
$$

If $\rho_{j}=0$, i.e., $\lambda_{j}=1$, the eigenvalues are $\bar{e}_{\alpha}=1 / 4$ for all $\alpha$. Thus, the effective dimension $\operatorname{dim}(E)=4$ and the space-time corresponds to an rectangular Euclidian space, i.e. a coordinate system for a resting particle. For a moving particle one obtains $\rho_{j}>0$, i.e., $\lambda_{j}<1$ leading to $\operatorname{dim}(E)<4$. Inserting Equation (14) into Equation (18) gives

$$
\begin{aligned}
& 4 \bar{e}_{0}=\breve{e}_{0}=2(1+\rho)(1+\lambda)^{-1}=2\left(1+\frac{2 \sqrt{1-Q}}{2-Q}\right)\left(1+\frac{Q}{2-Q}\right)^{-1}=\frac{2(2-Q+2 \sqrt{1-Q})}{2}=2-Q+2 \sqrt{1-Q} \\
& 4 \bar{e}_{1}=\breve{e}_{1}=2(1-\rho)(1+\lambda)^{-1}=2\left(1-\frac{2 \sqrt{1-Q}}{2-Q}\right)\left(1+\frac{Q}{2-Q}\right)^{-1}=\frac{2(2-Q-2 \sqrt{1-Q})}{2}=2-Q-2 \sqrt{1-Q} \\
& 4 \bar{e}_{2}=\breve{e}_{2}=4 \bar{e}_{3}=\breve{e}_{3}=2 \lambda /(1+\lambda)=\frac{2 Q}{2-Q}\left(1+\frac{Q}{2-Q}\right)^{-1}=2 Q(2-Q+Q)^{-1}=Q
\end{aligned}
$$


with $Q=q^{-1 / 2}=\left(E_{0} / E\right)^{2}=\left(m_{0} c^{2} / E\right)^{2}$ (note that the $x$-axis in Figure 3 presents $q=E / E_{0}$, ) and $\max (Q)=$ $\min (q)=1$. For the usual light elementary particles with masses equivalent to the GeV-energy scale, one obtains for the LES $\min (Q)=\left(m_{0} c^{2} / E_{P}\right)^{2}$, i.e. $\max (q)=\left(E_{P} / m_{0} c^{2}\right)^{2}$ and therefore, $\bar{e}_{0} \sim 1$ and $\bar{e}_{\alpha} \sim 0$ for $\alpha>0$. Next, one gets following expression for the entropy $S_{N}$ according to Equation (16)

$$
S_{N}(\varphi)=-\frac{k_{B}}{4} \sum \breve{e}_{\alpha} \log \left(\breve{e}_{\alpha} / 4\right)=-\frac{k_{B}}{4} \sum \breve{e}_{\alpha}\left[\log \left(\breve{e}_{\alpha}\right)-\log (4)\right]=k_{B} \log (4)-\frac{k_{B}}{4} \sum \breve{e}_{\alpha} \log \left(\breve{e}_{\alpha}\right)
$$

or

$$
\begin{aligned}
\frac{S_{N}(Q)}{k_{B}}= & \ln (4)-\frac{Q}{2} \ln (Q)-\frac{2-Q+2 \sqrt{1-Q}}{4} \ln (2-Q+2 \sqrt{1-Q}) \\
& +\frac{2-Q-2 \sqrt{1-Q}}{4} \times \ln (2-Q-2 \sqrt{1-Q})
\end{aligned}
$$

In Figure 3 the shapes of the effective dimensions, $\operatorname{dim}(E)$, versus the energy ratio, $\mathrm{E}_{\mathrm{E}} \mathrm{E}_{0}$, for three scalar particles with different rest energies are shown. The

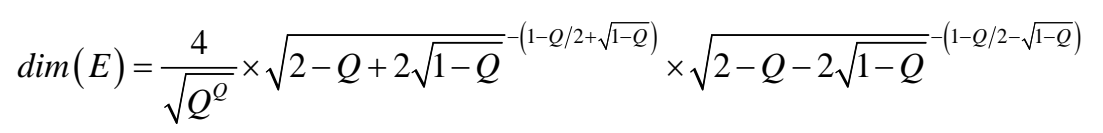

For the boundary region of LES one finds that $\left.\operatorname{dim}(E)\right|_{Q \sim 0} \sim 1$ (note: $\lim _{Q \rightarrow 0}\left(Q^{Q}\right)=1$ ) which corresponds to $\left.\operatorname{dim}(E)\right|_{q \sim q^{*}} \sim 1$ where $q^{*}=E_{P} / E_{0}$ (see Figure 3). To facilitate a comparison of the shape of $\operatorname{dim}(E)$ for a particle with Higgs mass (left plot of Figure 3 ) with that of hypothetical super-heavy particles the other two plots are presented. The soft decrease in the middle and right plot of Figure 3 results from the much higher masses of these hypothetical particles.

In contrast to the shapes of $\operatorname{dim}(E)$ for the super-heavy particles, the information loss calculated for the particle with Higgs mass reaches its limit $\operatorname{dim}(E)=1$ for an increasing energy very rapidly. Nevertheless, a comparison of the magnitudes of $q$ in the three plots shows, that the different shapes become similar to each other if the number of magnitudes would be the same in all plots of Figure 3. As a consequence, $\operatorname{dim}(E)$ differs between particles of different mass while $\operatorname{dim}(q)$ is a universal parameter. If Lorentz symmetry exists and the light speed is constant for the complete LES, the total energy of this sector cannot be higher than the Planckian limit, $m_{P} C^{2}$. Note, that in the left plot of Figure 3 the maximum energy is $\max (E) \sim 7 \times 10^{5} E_{H}$ (with $E_{H}=m_{H} c^{2}$ ) and much smaller than the maximum energy in the middle and right plots (with $\max (E) \sim E_{P}$ ). For an off-shell Higgs particle the dimensional limit is reached long before the quantum fluctuations of the metric become important, the Lorentz symmetry is intact and the LT is applicable even if $\operatorname{dim}(E) \approx 1$. For off-shell super-heavy particles,

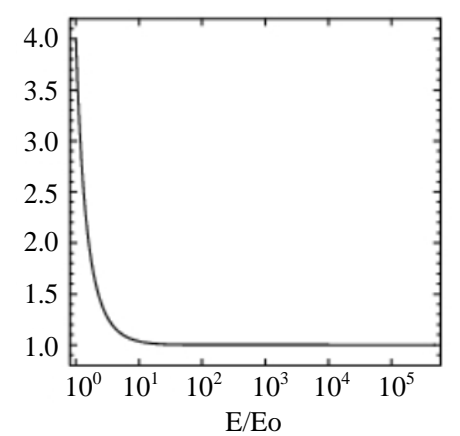

$\mathrm{E}_{0}=1.26 \times 10^{2} \mathrm{GeV}$

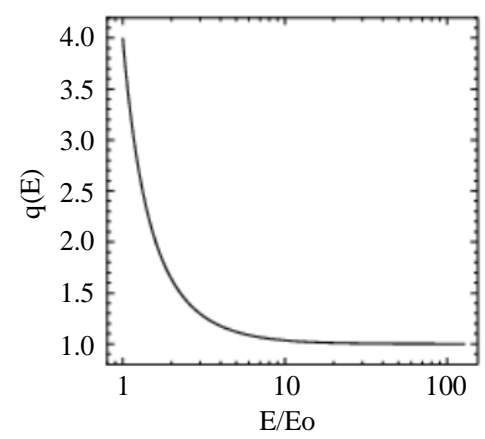

$\mathrm{E}_{0}=9.50 \times 10^{16} \mathrm{GeV}$

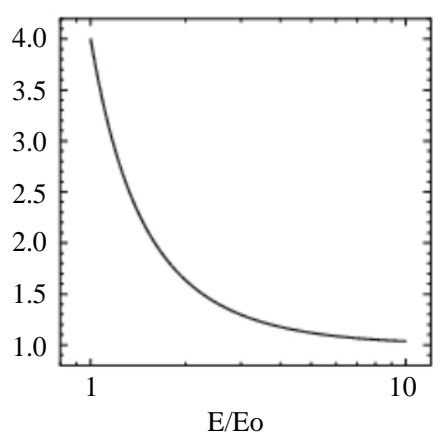

$\mathrm{E}_{0}=1.20 \times 10^{18} \mathrm{GeV}$

Figure 3. Loss of information, given by the dimension $\operatorname{dim}(E)$, between two corresponding inertial systems for a measuring device and a particle. This loss is shown for three particles with different masses in individual plots. Since $\operatorname{dim}(E)$ depends on the particle energy, an energy ratio, $q=E / E_{0}$ is introduced. The rest energy, $E_{0}=m_{0} c^{2}$, in the left plot results from the Higgs mass [1], $m_{0}=m_{H}$. In the middle and right plot, $E_{0}$ corresponds to the mass of hypothetical super-heavy particles close to the Planckian scale. The minimum energy $E$ on the x-axis of each plot varies, since the rest masses differ, $\operatorname{but} \min (q)=1$. The upper boundary value of $q$ is $q^{*}=E_{p} / E_{0}$ and varies in its value due to the different particle masses. 
especially for a particle with a mass $m_{0} \sim m_{P} / 10$ (right plot of Figure 3 ) the quantum fluctuations of the metric have started already while $\operatorname{dim}(E)>1$ and the transformation between inertial systems is not allowed anymore due to the broken Lorentz symmetry.

In the Introduction a transition region between the sector of the standard model is mentioned. For the simple scalar field this transition region needs not to be considered. However, the shapes of $\operatorname{dim}(\mathrm{E})$ for the super-heavy particles suggest the existence of some transition region.

\section{The Interacting Scalar Particle in the NoNRES}

Now, the derived integration measure is used for an interacting scalar quantum field, $\varphi$. We consider the 4-momentum $p_{\mu}$ of a free scalar particle and calculate the energy-momentum relation in the NoNRES

$$
g^{\tau v} p_{\tau} p_{v}=m_{0}^{2} g^{\tau v} u_{\tau} u_{v}=m_{0}^{2} c^{2}
$$

which leads to the Klein-Gordon operator $\aleph=\partial_{\alpha} \partial^{\alpha}+1$ where the inverse Compton length $l=m_{0} \cdot c / \hbar$ is used to scale the field $\varphi \rightarrow \varphi / l$ and the axes $x_{\mu} \rightarrow l x_{\mu}$. For a self-interacting scalar field, $\varphi$, the action integral $A[\varphi, J]$ is given by

$$
A[\varphi, J]=\int \mathrm{d}^{\operatorname{dim}} x \sqrt{g}\left[-\varphi(x)\left(\partial^{\alpha} \partial_{\alpha}+1\right) \varphi(x) / 2-V[\varphi]+J(x) \varphi(x)\right]
$$

where the potential term is $V[\varphi]=w / 4 ! \cdot \varphi^{4}, J$ is some background field and $\operatorname{dim}$ is the dimension of the particle related space-time. In contrast to the MS, the metric factor $g^{1 / 2}$ of the NoNRES, occurring in the action, gives 1 . Accordingly, the path integral can be written as $\exp (-A / \hbar)$, unlike $\exp (-i A / \hbar)$ in the MS. The Green's function, $D(x)$, of a free scalar field with $V[\varphi]=0$ can be calculated from

$$
D(x)=\frac{1}{(2 \pi)^{4}} \int \mathrm{d}^{\mathrm{dim}} k \frac{\exp (-i x k)}{k^{2}-1+i \varepsilon} \Leftrightarrow\left(\partial^{\alpha} \partial_{\alpha}+1\right) D(x)=\aleph D(x)=-\delta(x)
$$

where $k_{v} \rightarrow k_{\nu} / l$ and $i \varepsilon$ is a regularization term for the denominator if $k^{2}=1$. The connected 2-point Green's function (compare to the dressed propagator in Figure 1) for the weakly self-interacting field in the one-loop approximation is given by

$$
\Delta\left(x_{1}, x_{2}\right)=D\left(x_{1}-x_{2}\right)+\frac{\hbar w}{2} D(0) \int \mathrm{d}^{\operatorname{dim}} z D\left(z-x_{1}\right) D\left(z-x_{2}\right) .
$$

The Fourier transform of Equation (22) can be expressed as

$$
\Gamma(q,-q)=\left(q^{2}-1+i \varepsilon\right)^{-1}+\frac{\hbar w}{2}\left(q^{2}-1+i \varepsilon\right)^{-1} \int \frac{d^{d i m} k}{(2 \pi)^{4}}\left(k^{2}-1+i \varepsilon\right)^{-1}\left(q^{2}-1+i \varepsilon\right)^{-1} .
$$

Since the interaction given by Equation $(20)$ is sufficiently weak $(w \hbar D(x=0) \ll 1)$ one can write for Equation (23)

$$
\Gamma(q,-q)=\frac{1}{q^{2}-\{1+w \hbar / 2 \cdot \mathfrak{R}[D(0)]\}+i\{\varepsilon-w \hbar / 2 \cdot \mathfrak{I}[D(0)]\}}
$$

where the scaled corrected mass and lifetime of the weakly self-interacting field, $\varphi$, result from

$$
\mu^{*}=\sqrt{1+w \hbar \Re[D(0)] / 2} \text { and } \tau_{L}^{*}=\sqrt{2 /\{w \hbar \mathfrak{I}[D(0)]\}} .
$$

In the MS $D(0)$ is UV divergent. However, in the NoNRES we obtain a finite expression as shown below. Considering $\operatorname{dim}=\operatorname{dim}(E)$ the integral for $D(0)$ in Equation (23), which depends on $k^{2}$ only, is given by

$$
D(0)=\int \mathrm{d} k \frac{2 k^{\operatorname{dim}(E)-1}\left(k^{2}-1+i \varepsilon\right)^{-1}}{(4 \pi)^{\operatorname{dim}(E) / 2} \Gamma[q(E) / 2]} \stackrel{\operatorname{dim}=4}{\longrightarrow} D(0)=\frac{2}{(4 \pi)^{2} \Gamma[2]} \int \frac{\mathrm{d} k k^{3}}{k^{2}-1+i \varepsilon}
$$


where the integration range of $\mathrm{k}$ extends from null to infinity. From this equation the sought integration measure, which is $\mathrm{d}^{\operatorname{dim}(E)} k$, can be read off. In the special case of a $k^{2}$-dependent integration function, it simplifies to $\mathrm{d}^{\operatorname{dim}(E)} k=\zeta(\operatorname{dim}) \mathrm{d} k k^{\operatorname{dim}(E)-1}$. The integration range is split into the two energy sectors as explained in the Introduction. They go from the $k=0$ to $E_{p} / m_{0} c^{2}$ and from $k=E_{p} / m_{0} c^{2}$ to $\infty$. If the energy $E<E_{P}$ the physical space is modeled by a 4-dimensional rectangular Euclidean space due to the intact Lorentz symmetry and the applicability of the corresponding LT. If $E \sim E_{P}$ the LT is not allowed anymore. However, $\operatorname{dim}(E)$ has reached the limit $\operatorname{dim}(E)=1$ long before (see Figure 3), thus, we can find a jump function for the effective dimension of the local NoNRES written as

$$
\operatorname{dim}(E)=4 \text { if } E<E_{P} \text { and } \operatorname{dim}(E)=1 \text { if } E \geq E_{P} .
$$

Consequently, we obtain for Equation (26)

$$
D(0)=\frac{1}{8 \pi^{2}} \int_{0}^{k^{*}} \frac{\mathrm{d} k k^{3}}{k^{2}-1+i \varepsilon}+\frac{1}{\pi} \int_{k^{*}}^{\infty} \frac{\mathrm{d} k}{k^{2}-1+i \varepsilon} .
$$

Both terms are finite and give

$$
D(0)=\frac{1}{16 \pi^{2}}\left(k^{* 2}+\ln \left(k^{* 2}-1\right)-i \pi\right)+\frac{1}{\pi}\left(\arctan \left(k^{*}\right)+i \pi / 2\right) \approx \frac{k^{* 2}}{16 \pi^{2}}+0.48 i .
$$

Obviously, the effective dimension, $\operatorname{dim}(E)$, acts as a "regularization-like parameter" influencing the integration result in the high-energy range. However, in contrast to usual regularization procedures in the MS, $\operatorname{dim}(E)$ leads to a convergent result without specific normalization concepts.

Because of scaling we substitute for $k^{*}$ the ratio of Planckian to particle rest energy, i.e., $k^{*}=E_{P} / m_{0} c^{2}$. Since the generator of the massive scalar field, $\varphi$, is assumed to be the Higgs particle, it results $m_{0} c^{2} \equiv m_{H} c^{2}=126 \mathrm{GeV}$ [1] [2], where $m_{H}$ is its mass and $c$ is the light speed, as has already been used to calculate the shape of $\operatorname{dim}(E)$ presented in Figure 3. Therefore, we obtain for the corrected mass, $\mu^{*}$, and the un-scaled lifetime, $\tau_{L}$, derived from Equation (25), the following results

$$
\begin{gathered}
\mu^{*}=\sqrt{1+w \hbar \Re(D(0)) / 2} \approx \sqrt{1+1.4 \times 10^{-3}(\mathrm{w} \cdot \mathrm{Js})} \\
\tau_{L}=\hbar \tau_{L}^{*} /\left(m_{0} c^{2}\right)=\sqrt{2 \hbar / w \mathfrak{I}(D(0))}\left(m_{0} c^{2}\right)^{-1} \approx 0.69(\mathrm{w} \cdot \mathrm{Js})^{-1 / 2} \mathrm{~ns}
\end{gathered}
$$

The coupling constant should be $w \leq 10 / \mathrm{Js}$ to guarantee the applicability of a first order approximation to re-arrange Equation (23). If the particle mass is not the estimated Higgs mass, but, e.g., the electron mass, we had to require a much lower upper boundary for $w$ or Equation (24) would be invalid. The theory described by Equation (20) is true for massive scalar particles. If one likes to consider massless particles, e.g. photons, together with Higgs scalars, the standard model of the electro-weak and strong interaction needs to be slightly modified to ensure its Lorentz invariance in the NoNRES.

\section{Conclusions}

The metric, $g^{\mu v}$, in the NoNRES, which depends on the particle energy, $E$, allows to derivate an effective dimension, $\operatorname{dim}(E)$, which is the key parameter of the integration measure $\mathrm{d}^{\operatorname{dim}(E)} k$. In contrast to the NoNRES, the effective dimension of the MS always gives $\operatorname{dim}_{\mathrm{MS}}(E)=4$ for all values of $E$, i.e., it is independent of the particle energy. The described integration-method constructed for the NoNRES involves the high energy range of the specific QFT more appropriately than the MS, since the available information content of the observed process is considered and quantified. In addition, the NoNRES can be constructed for space-times of arbitrary dimensions. The results obtained for Equations (30) and (31) are quite reasonable. In contrast to the mass correction, the life time of the particle is mainly influenced by the result of the high energy sector, i.e., the large imaginary part of $D(0)$ in Equation (29).

In this study a self-interacting scalar particle is considered. In order to develop a real QFT in the NoNRES we have to include particles with a larger coupling constant (i.e. higher order loop integrals need to be taken into account) and particles with a spin larger than 0 . Then, QED (quantum electrodynamics) can be studied to model 
the interaction between electrons by means of photon exchange. In comparison to Equation (20), the QED action integral is more complex and contains the Abelian gauge field, $A_{\alpha}$, and a spinor field, $\psi$. An extension of our integration method to QED would open the access to real measurements.

\section{Acknowledgements}

The author would like to cordially thank Uwe Motschmann from the Institute for Theoretical Physics at the Technical University Braunschweig for many fruitful discussions.

\section{References}

[1] Aad, G., et al. [ATLAS Collaboration] (2012) Physics Letters B, 716, 1-29. http://dx.doi.org/10.1016/j.physletb.2012.08.020

[2] Chatrchyan, S., et al. [CMS Collaboration] (2012) Physics Letters B, 716, 30-61. http://dx.doi.org/10.1016/j.physletb.2012.08.02s1

[3] Delamotte, B. (2004) American Journal of Physics, 72, 170-184. http://dx.doi.org/10.1119/1.1624112

[4] Erdmenger, J., Meyer, R. and Park, J.-H. (2007) Physical Review Letters, 98, Article ID: 261301. http://dx.doi.org/10.1103/PhysRevLett.98.261301

[5] Gharibyan, V. (2012) Physical Review Letters, 109, Article ID: 141103. http://dx.doi.org/10.1103/PhysRevLett.109.141103

[6] Balian, R. (2003) Séminaire Poincaré, 2, 13-27.

[7] Twomey, S. (2002) Introduction to the Mathematics of Inversion in Remote Sensing and Indirect Measurements, Courier Dover Publications. 
Scientific Research Publishing (SCIRP) is one of the largest Open Access journal publishers. It is currently publishing more than 200 open access, online, peer-reviewed journals covering a wide range of academic disciplines. SCIRP serves the worldwide academic communities and contributes to the progress and application of science with its publication.

Other selected journals from SCIRP are listed as below. Submit your manuscript to us via either submit@scirp.org or Online Submission Portal.
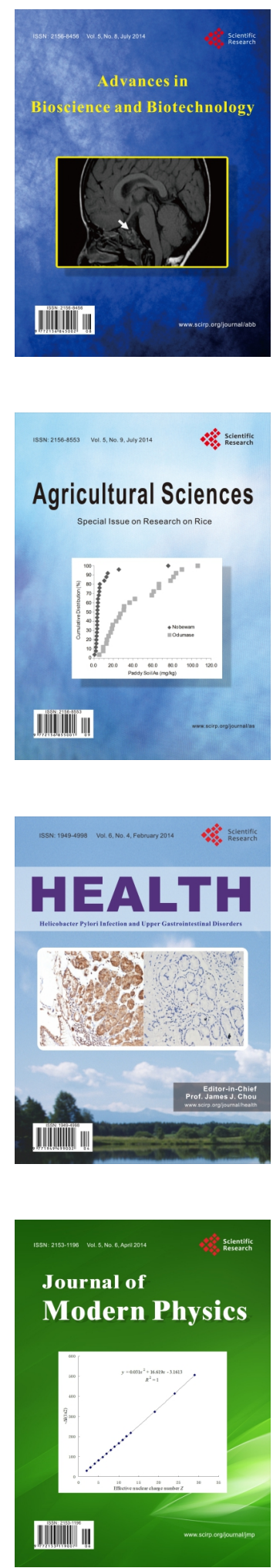
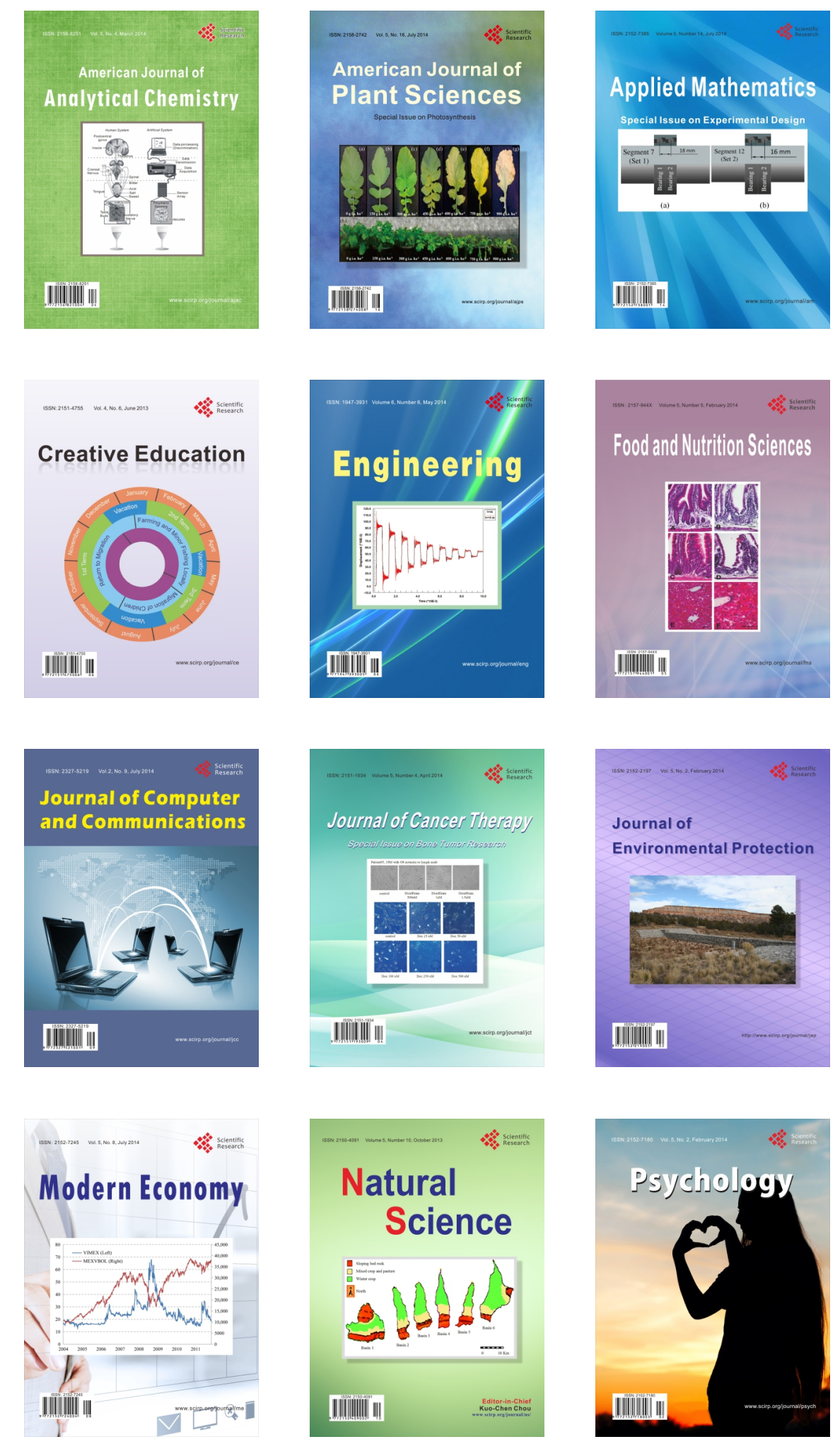